\title{
Stable Shape Comparison of Surfaces via Reeb Graphs
}

\author{
Barbara Di Fabio ${ }^{1,3}$ and Claudia Landi ${ }^{2,3}$ \\ 1 Dipartimento di Matematica, Università di Bologna, Italy \\ barbara.difabio@unibo.it \\ 2 DISMI, Università di Modena e Reggio Emilia, Italy \\ claudia.landi@unimore.it \\ 3 ARCES, Università di Bologna, Italy
}

\begin{abstract}
Reeb graphs are combinatorial signatures that capture shape properties from the perspective of a chosen function. One of the most important questions is whether Reeb graphs are robust against function perturbations that may occur because of noise and approximation errors in the data acquisition process. In this work we tackle the problem of stability by providing an editing distance between Reeb graphs of orientable surfaces in terms of the cost necessary to transform one graph into another by edit operations. Our main result is that the editing distance between two Reeb graphs is upper bounded by the extent of the difference of the associated functions, measured by the maximum norm. This yields the stability property under function perturbations.
\end{abstract}

Keywords: Shape similarity, editing distance, Morse function.

\section{Introduction}

In shape comparison, a widely used scheme is to measure the dissimilarity between signatures associated with each shape rather than match shapes directly [141218].

Reeb graphs are signatures describing shapes from topological and geometrical perspectives. In this framework, shapes are modeled as spaces $X$ endowed with scalar functions $f$. The role of $f$ is to explore geometrical properties of the space $X$. The Reeb graph of $f: X \rightarrow \mathbb{R}$ is obtained by shrinking each connected component of a level set of $f$ to a single point [15.

Reeb graphs have been used as an effective tool for shape analysis and description tasks since [17/6]. The Reeb graph has a number of characteristics that make it useful as a search key for 3D objects. First, a Reeb graph always consists of a one-dimensional graph structure and does not have any higher dimension components such as the degenerate surface that can occur in a medial axis. Second, by defining the function appropriately, it is possible to construct a Reeb graph that is invariant to translation and rotation, or even more complicated isometries of the shape. 
One of the most important questions is whether Reeb graphs are robust against perturbations that may occur because of noise and approximation errors in the data acquisition process. Heuristics have been developed so that the Reeb graph turns out to be resistant to connectivity changes caused by simplification, subdivision and remesh, and robust against noise and certain changes due to deformation 104 .

In this paper we tackle the robustness problem for Reeb graphs from a theoretical point of view. The main idea is to generalize to the case of surfaces the techniques developed in [6] to prove the stability of Reeb graphs of curves against function perturbations. Indeed the case of surfaces appears as the most interesting area of applications of the Reeb graph as a shape descriptor.

To this end, we introduce a combinatorial dissimilarity measure, called an editing distance, between Reeb graphs of surfaces in terms of the cost necessary to transform one graph into another by edit operations. Thus our editing distance between Reeb graphs belongs to the family of Graph Edit Distances [9], widely used in pattern analysis. As shown in [9], some of these Graph Edit Distances are metrics, some other are only pseudo-metrics. Our editing distance turns out to have all the properties of a pseudo-metric. The main result we provide is that the editing distance between two Reeb graphs is never greater than the extent of the difference of the associated functions, measured by the maximum norm, yielding the stability property under function perturbations.

In the literature, some other comparison methodologies have been proposed to compare Reeb graphs and estimate the similarity of the shapes described by Reeb graph.

In 10] the authors propose a Multiresolutional Reeb Graph (MRG) based on geodesic distance. Similarity between 3D shapes is calculated using a coarse-tofine strategy while preserving the topological consistency of the graph structures to provide a fast and efficient estimation of similarity and correspondence between shapes.

In [4] the authors discuss a method for measuring the similarity and recognizing sub-part correspondences of 3D shapes, based on the synergy of a structural descriptor, like the Extended Reeb Graph, with a geometric descriptor, like spherical harmonics.

Although the matching frameworks proposed in [10] and [4 are characterized by several computational advantages, the methods provided for Reeb graphs comparison have not been proved to be stable with respect to noise in the data, differently from the method proposed here.

Only recently the problem of Reeb graph stability has been investigated from the theoretical point of view.

In [6] an editing distance between Reeb graphs of curves endowed with Morse functions is introduced and shown to yield stability. Importantly, despite the combinatorial nature of this distance, it coincides with the natural pseudodistance between shapes [8], thus showing the maximal discriminative power for this sort of distances. 
The work in [2] about a stable distance for merge trees is also pertinent to the stability problem for Reeb graphs: merge trees are known to determine contour trees, that are Reeb graphs for simple domains.

Recently a functional distortion distance between Reeb graphs has been proposed in the preprint [1], with proven stable and discriminative properties. The functional distortion distance is intrinsically continuous, whereas the editing distance we propose is combinatorial.

In conclusion, the novelty of this paper is the announcement of a new combinatorial method to compare Reeb graphs in a stable way when shapes can be modeled as surfaces. An outline of the proof of this stability result is also given here, while full details and technicalities can be found in the technical report 7 .

Outline. Section 2 reviews Reeb graphs. Section 3 introduces the admissible editing deformations to transform Reeb graphs into each other. In Section 4 the editing distance is defined. Section 5 illustrates the stability of Reeb graphs with respect to the editing distance. Section 6 concludes the paper.

\section{Preliminaries on Reeb Graphs}

An overview of the properties of Reeb graphs from the mathematical foundations to its history in the Computer Graphics context can be found in [3].

Since the main focus of this paper is on theoretical aspects, and computational issues being postponed to a future research, the appropriate setting for studying Reeb graphs is the following one.

$\mathcal{M}$ is a smooth (i.e. differentiable of class at least $C^{2}$ ) closed (i.e. compact and without boundary) orientable surface, and $f: \mathcal{M} \rightarrow \mathbb{R}$ is a simple Morse function on $\mathcal{M}$, i.e., a smooth function such that its Hessian matrix at each critical point is non-singular and, for every two distinct critical points $p$ and $q$ of $f$, the associated critical level sets $f^{-1}(f(p))$ and $f^{-1}(f(q))$ are disjoint.

Definition 1. For every $p, q \in \mathcal{M}$, set $p \sim q$ whenever $p, q$ belong to the same connected component of $f^{-1}(f(p))$. The quotient space $\mathcal{M} / \sim$ is a finite and connected simplicial complex of dimension 1 known as the Reeb graph associated with $f$.

Hence, the Reeb graph of a simple Morse function $f: \mathcal{M} \rightarrow \mathbb{R}$ is a graph whose vertices are the connected components of the critical levels of $f$ that contain a critical point.

The Reeb graph associated with $f$ will be denoted by $\Gamma_{f}$, its vertex set by $V\left(\Gamma_{f}\right)$, and its edge set by $E\left(\Gamma_{f}\right)$. Moreover, if $v_{1}, v_{2} \in V\left(\Gamma_{f}\right)$ are adjacent vertices, i.e., connected by an edge, we will write $e\left(v_{1}, v_{2}\right) \in E\left(\Gamma_{f}\right)$.

The critical points of $f$ correspond bijectively to the vertices of $\Gamma_{f}$. In particular, the assumption that $\mathcal{M}$ is orientable ensures that the vertices of $\Gamma_{f}$ can be either of degree 1 (when corresponding to minima or maxima of $f$ ), or of degree 3 (when corresponding to saddles of $f$ ). Moreover, if $\mathcal{M}$ has genus $\mathfrak{g}, \Gamma_{f}$ has exactly $\mathfrak{g}$ linearly independent cycles. 
In what follows, we label each vertex of $\Gamma_{f}$ by the value taken by $f$ at the corresponding critical point. We denote such a labeled graph by $\left(\Gamma_{f}, \ell_{f}\right)$, where $\ell_{f}: V\left(\Gamma_{f}\right) \rightarrow \mathbb{R}$ is the restriction of $f: \mathcal{M} \rightarrow \mathbb{R}$ to the set of its critical points. In a labeled Reeb graph, each vertex $v$ of degree 3 has at least two of its adjacent vertices, say $w, w^{\prime}$, such that $\ell_{f}(w)<\ell_{f}(v)<\ell_{f}\left(w^{\prime}\right)$. An example is displayed in Figure 1.

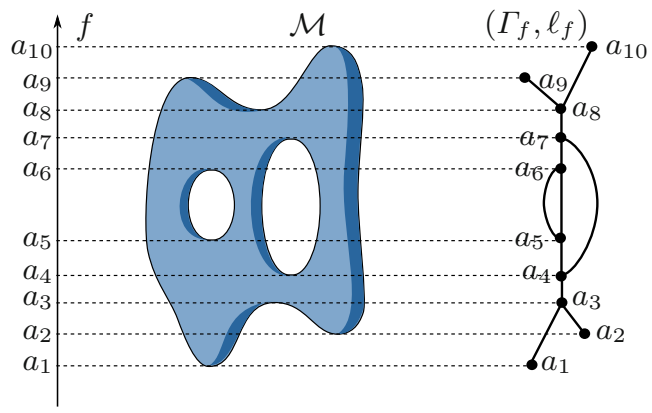

Fig. 1. Left: the height function $f: \mathcal{M} \rightarrow \mathbb{R}$; center: the surface $\mathcal{M}$ of genus $\mathfrak{g}=2$; right: the associated labeled Reeb graph $\left(\Gamma_{f}, \ell_{f}\right)$

Following [13], it can be seen that, given a graph on an even number of vertices, all of which are of degree 1 or 3, appropriately labeled, there is a simple Morse function whose labeled Reeb graph is the given one. This result requires the following definition.

Definition 2. We shall say that two labeled Reeb graphs $\left(\Gamma_{f}, \ell_{f}\right),\left(\Gamma_{g}, \ell_{g}\right)$ are isomorphic, and we write $\left(\Gamma_{f}, \ell_{f}\right) \cong\left(\Gamma_{g}, \ell_{g}\right)$, if there exists a graph isomorphism $\Phi: V\left(\Gamma_{f}\right) \rightarrow V\left(\Gamma_{g}\right)$ such that, for every $v \in V\left(\Gamma_{f}\right), f(v)=g(\Phi(v)$ ) (i.e. $\Phi$ preserves edges and vertices labels).

Proposition 1 (Realization theorem). Let $(G, \ell)$ be a labeled graph, where $G$ is a graph with $m$ linearly independent cycles, on an even number of vertices, all of which are of degree 1 or 3 , and $\ell: V(G) \rightarrow \mathbb{R}$ is an injective function such that, for any vertex $v$ of degree 3, at least two among its adjacent vertices, say $w, w^{\prime}$, are such that $\ell(w)<\ell(v)<\ell\left(w^{\prime}\right)$. Then an orientable closed surface $\mathcal{M}$ of genus $\mathfrak{g}=m$, and a simple Morse function $f: \mathcal{M} \rightarrow \mathbb{R}$ exist such that $\left(\Gamma_{f}, \ell_{f}\right) \cong(G, \ell)$.

One may wonder if such surface and function are also unique, up to labeled graph isomorphism. Following [7, we answer to this question by considering two equivalence relations on the space of functions, and studying how they are mirrored by Reeb graphs isomorphisms.

Definition 3. Let $\mathcal{D}(\mathcal{M})$ be the set of self-diffeomorphisms of $\mathcal{M}$. Two simple Morse functions $f, g: \mathcal{M} \rightarrow \mathbb{R}$ are called right-equivalent if there exists $\xi \in$ 
$\mathcal{D}(\mathcal{M})$ such that $f=g \circ \xi$. Moreover, $f, g$ are called right-left equivalent if there exist $\xi \in \mathcal{D}(\mathcal{M})$ and an orientation preserving self-diffeomorphism $\eta$ of $\mathbb{R}$ such that $f=\eta \circ g \circ \xi$.

Proposition 2 (Uniqueness theorem). If $f, g$ are simple Morse functions on a closed surface, then

1. $f$ and $g$ are right-left equivalent if and only if their Reeb graphs $\Gamma_{f}$ and $\Gamma_{g}$ are isomorphic by an isomorphism that preserves the vertex order;

2. $f$ and $g$ are right-equivalent if and only if their labeled Reeb graphs $\left(\Gamma_{f}, \ell_{f}\right)$ and $\left(\Gamma_{g}, \ell_{g}\right)$ are isomorphic.

\section{$3 \quad$ Editing Deformations}

In this section we present the moves that allow to edit Reeb graphs into each other. Basically, these moves amount to finite ordered sequences of elementary deformations.

Elementary deformations allow us to transform a Reeb graph into another with either a different number of vertices (birth (B) and death (D)), or with the same number of vertices endowed with different labels (relabeling $(\mathrm{R})$ and moves by Kudryavtseva $\left.\left(\mathrm{K}_{1}\right),\left(\mathrm{K}_{2}\right),\left(\mathrm{K}_{3}\right)[1]\right)$. We underline that the definition of the deformations of type (B), (D) and (R) is essentially different from the definition of analogous deformations in the case of Reeb graphs of curves as given in [6], even if the associated cost will be the same (see Section 4). This is because the degree of the involved vertices is 2 for Reeb graphs of closed curves, 1 and 3 for Reeb graphs of surfaces.

Definition 4. With the convention of denoting the open interval with endpoints $a, b$ by $] a, b\left[\right.$, the elementary deformations $(\mathrm{B}),(\mathrm{D}),(\mathrm{R}),\left(\mathrm{K}_{i}\right), i=1,2,3$, can be defined as follows.

(B) For a fixed edge $e\left(v_{1}, v_{2}\right) \in E\left(\Gamma_{f}\right)$, with $\ell_{f}\left(v_{1}\right)<\ell_{f}\left(v_{2}\right), T$ is an elementary deformation of $\left(\Gamma_{f}, \ell_{f}\right)$ of type $(\mathrm{B})$ if $T\left(\Gamma_{f}, \ell_{f}\right)$ is a labeled Reeb graph $\left(\Gamma_{g}, \ell_{g}\right)$ such that

- $V\left(\Gamma_{g}\right)=V\left(\Gamma_{f}\right) \cup\left\{u_{1}, u_{2}\right\}$;

- $E\left(\Gamma_{g}\right)=\left(E\left(\Gamma_{f}\right)-\left\{e\left(v_{1}, v_{2}\right)\right\}\right) \cup\left\{e\left(v_{1}, u_{1}\right), e\left(u_{1}, u_{2}\right), e\left(u_{2}, v_{2}\right)\right\}$;

- $\ell_{f}\left(v_{1}\right)<\ell_{g}\left(u_{i}\right)<\ell_{g}\left(u_{j}\right)<\ell_{f}\left(v_{2}\right)$, with $\ell_{g}^{-1}(] \ell_{g}\left(u_{i}\right), \ell_{g}\left(u_{j}\right)[)=\emptyset, i, j \in$ $\{1,2\}, i \neq j$, and $\ell_{g \mid V\left(\Gamma_{f}\right)}=\ell_{f}$.

(D) For fixed edges $e\left(v_{1}, u_{1}\right), e\left(u_{1}, u_{2}\right), e\left(u_{1}, v_{2}\right) \in E\left(\Gamma_{f}\right), u_{2}$ being of degree 1 , such that $\ell_{f}\left(v_{1}\right)<\ell_{f}\left(u_{i}\right)<\ell_{f}\left(u_{j}\right)<\ell_{f}\left(v_{2}\right)$, with $\ell_{f}^{-1}(] \ell_{f}\left(u_{i}\right), \ell_{f}\left(u_{j}\right)[)=$ $\emptyset, i, j \in\{1,2\}, i \neq j, T$ is an elementary deformation of $\left(\Gamma_{f}, \ell_{f}\right)$ of type (D) if $T\left(\Gamma_{f}, \ell_{f}\right)$ is a labeled Reeb graph $\left(\Gamma_{g}, \ell_{g}\right)$ such that

- $V\left(\Gamma_{g}\right)=V\left(\Gamma_{f}\right)-\left\{u_{1}, u_{2}\right\}$;

- $E\left(\Gamma_{g}\right)=\left(E\left(\Gamma_{f}\right)-\left\{e\left(v_{1}, u_{1}\right), e\left(u_{1}, u_{2}\right), e\left(u_{2}, v_{2}\right)\right\}\right) \cup\left\{e\left(v_{1}, v_{2}\right)\right\}$;

- $\ell_{g}=\ell_{f \mid V\left(\Gamma_{f}\right)-\left\{u_{1}, u_{2}\right\}}$. 
(R) $T$ is an elementary deformation of $\left(\Gamma_{f}, \ell_{f}\right)$ of type $(\mathrm{R})$ if $T\left(\Gamma_{f}, \ell_{f}\right)$ is a labeled Reeb graph $\left(\Gamma_{g}, \ell_{g}\right)$ such that

- $\Gamma_{g}=\Gamma_{f}$;

- $\ell_{g}: V(G) \rightarrow \mathbb{R}$ induces the same vertex-order as $\ell_{f}$ except for at most two non-adjacent vertices, say $u_{1}, u_{2}$, for which, if $\ell_{f}\left(u_{1}\right)<\ell_{f}\left(u_{2}\right)$ and $\ell_{f}^{-1}(] \ell_{f}\left(u_{1}\right), \ell_{f}\left(u_{2}\right)[)=\emptyset$, then $\ell_{g}\left(u_{1}\right)>\ell_{g}\left(u_{2}\right)$, and $\ell_{g}^{-1}(] \ell_{g}\left(u_{2}\right)$, $\ell_{g}\left(u_{1}\right)[)=\emptyset$.

$\left(\mathrm{K}_{1}\right)$ For fixed edges $e\left(v_{1}, u_{1}\right), e\left(u_{1}, u_{2}\right), e\left(u_{1}, v_{4}\right), e\left(u_{2}, v_{2}\right), e\left(u_{2}, v_{3}\right) \in E\left(\Gamma_{f}\right)$, with two among $v_{2}, v_{3}, v_{4}$ possibly coincident, and either $\ell_{f}\left(v_{1}\right)<\ell_{f}\left(u_{1}\right)<$ $\ell_{f}\left(u_{2}\right)<\ell_{f}\left(v_{2}\right), \ell_{f}\left(v_{3}\right), \ell_{f}\left(v_{4}\right)$, with $\ell_{f}^{-1}(] \ell_{f}\left(u_{1}\right), \ell_{f}\left(u_{2}\right)[)=\emptyset$, or $\ell_{f}\left(v_{2}\right)$, $\ell_{f}\left(v_{3}\right), \ell_{f}\left(v_{4}\right)<\ell_{f}\left(u_{2}\right)<\ell_{f}\left(u_{1}\right)<\ell_{f}\left(v_{1}\right)$, with $\ell_{f}^{-1}(] \ell_{f}\left(u_{2}\right), \ell_{f}\left(u_{1}\right)[)=\emptyset$, $T$ is an elementary deformation of $\left(\Gamma_{f}, \ell_{f}\right)$ of type $\left(\mathrm{K}_{1}\right)$ if $T\left(\Gamma_{f}, \ell_{f}\right)$ is a labeled Reeb graph $\left(\Gamma_{g}, \ell_{g}\right)$ such that:

- $V\left(\Gamma_{g}\right)=V\left(\Gamma_{f}\right)$;

- $E\left(\Gamma_{g}\right)=\left(E\left(\Gamma_{f}\right)-\left\{e\left(v_{1}, u_{1}\right), e\left(u_{2}, v_{2}\right)\right\}\right) \cup\left\{e\left(v_{1}, u_{2}\right), e\left(u_{1}, v_{2}\right)\right\}$;

- $\ell_{g \mid V\left(\Gamma_{g}\right)-\left\{u_{1}, u_{2}\right\}}=\ell_{f}$, and either $\ell_{f}\left(v_{1}\right)<\ell_{g}\left(u_{2}\right)<\ell_{g}\left(u_{1}\right)<\ell_{f}\left(v_{2}\right)$, $\ell_{f}\left(v_{3}\right), \ell_{f}\left(v_{4}\right)$, with $\ell_{g}^{-1}(] \ell_{g}\left(u_{2}\right), \ell_{g}\left(u_{1}\right)[)=\emptyset$, or $\ell_{f}\left(v_{2}\right), \ell_{f}\left(v_{3}\right), \ell_{f}\left(v_{4}\right)<$ $\ell_{g}\left(u_{1}\right)<\ell_{g}\left(u_{2}\right)<\ell_{f}\left(v_{1}\right)$, with $\ell_{g}^{-1}(] \ell_{g}\left(u_{1}\right), \ell_{g}\left(u_{2}\right)[)=\emptyset$.

$\left(\mathrm{K}_{2}\right)$ For fixed edges $e\left(v_{1}, u_{1}\right), e\left(u_{1}, u_{2}\right), e\left(v_{2}, u_{1}\right), e\left(u_{2}, v_{3}\right), e\left(u_{2}, v_{4}\right) \in E\left(\Gamma_{f}\right)$, with $u_{1}, u_{2}$ of degree $3, v_{2}, v_{3}$ possibly coincident with $v_{1}, v_{4}$, respectively, and $\ell_{f}\left(v_{1}\right), \ell_{f}\left(v_{2}\right)<\ell_{f}\left(u_{1}\right)<\ell_{f}\left(u_{2}\right)<\ell_{f}\left(v_{3}\right), \ell_{f}\left(v_{4}\right)$, with $\ell_{f}^{-1}(] \ell_{f}\left(u_{1}\right)$, $\ell_{f}\left(u_{2}\right)[)=\emptyset, T$ is an elementary deformation of $\left(\Gamma_{f}, \ell_{f}\right)$ of type $\left(\mathrm{K}_{2}\right)$ if $T\left(\Gamma_{f}, \ell_{f}\right)$ is a labeled Reeb graph $\left(\Gamma_{g}, \ell_{g}\right)$ such that:

- $V\left(\Gamma_{g}\right)=V\left(\Gamma_{f}\right)$;

- $\left.E\left(\Gamma_{g}\right)=\left(E\left(\Gamma_{f}\right)-e\left(v_{1}, u_{1}\right), e\left(u_{2}, v_{3}\right)\right\}\right) \cup\left\{e\left(u_{1}, v_{3}\right), e\left(v_{1}, u_{2}\right)\right\}$;

- $\ell_{g \mid V\left(\Gamma_{g}\right)-\left\{u_{1}, u_{2}\right\}}=\ell_{f}$ and $\ell_{f}\left(v_{1}\right), \ell_{f}\left(v_{2}\right)<\ell_{g}\left(u_{2}\right)<\ell_{g}\left(u_{1}\right)<\ell_{f}\left(v_{3}\right)$, $\ell_{f}\left(v_{4}\right)$, with $\ell_{g}^{-1}(] \ell_{g}\left(u_{2}\right), \ell_{g}\left(u_{1}\right)[)=\emptyset$.

$\left(\mathrm{K}_{3}\right)$ For fixed edges $e\left(v_{1}, u_{2}\right), e\left(u_{1}, u_{2}\right), e\left(v_{2}, u_{1}\right), e\left(u_{1}, v_{3}\right), e\left(u_{2}, v_{4}\right) \in E\left(\Gamma_{f}\right)$, with $u_{1}, u_{2}$ of degree $3, v_{2}, v_{3}$ possibly coincident with $v_{1}, v_{4}$, respectively, and $\ell_{f}\left(v_{1}\right), \ell_{f}\left(v_{2}\right)<\ell_{f}\left(u_{2}\right)<\ell_{f}\left(u_{1}\right)<\ell_{f}\left(v_{3}\right), \ell_{f}\left(v_{4}\right)$, with $\ell_{f}^{-1}(] \ell_{f}\left(u_{2}\right)$, $\ell_{f}\left(u_{1}\right)[)=\emptyset, T$ is an elementary deformation of $\left(\Gamma_{f}, \ell_{f}\right)$ of type $\left(\mathrm{K}_{3}\right)$ if $T\left(\Gamma_{f}, \ell_{f}\right)$ is a labeled Reeb graph $\left(\Gamma_{g}, \ell_{g}\right)$ such that:

- $V\left(\Gamma_{g}\right)=V\left(\Gamma_{f}\right)$;

- $\left.E\left(\Gamma_{g}\right)=\left(E\left(\Gamma_{f}\right)-e\left(v_{1}, u_{2}\right), e\left(u_{1}, v_{3}\right)\right\}\right) \cup\left\{e\left(v_{1}, u_{1}\right), e\left(u_{2}, v_{3}\right)\right\}$;

- $\ell_{g \mid V\left(\Gamma_{g}\right)-\left\{u_{1}, u_{2}\right\}}=\ell_{f}$ and $\ell_{f}\left(v_{1}\right), \ell_{f}\left(v_{2}\right)<\ell_{g}\left(u_{1}\right)<\ell_{g}\left(u_{2}\right)<\ell_{f}\left(v_{3}\right)$, $\ell_{f}\left(v_{4}\right)$, with $\ell_{g}^{-1}(] \ell_{g}\left(u_{1}\right), \ell_{g}\left(u_{2}\right)[)=\emptyset$.

All the elementary deformations above defined are schematically displayed in Table 1 .

We observe that, differently from the case of curves [6], it is not sufficient to consider only deformations of type (B), (D) and (R). The necessity to add those of type $\left(\mathrm{K}_{i}\right), i=1,2,3$, can be in fact deduced by observing the changes a Reeb graph undergoes when it is dynamically associated with Morse functions that at a some instant fail to be simple (Table 11). Only in some particular cases, such as when some of the vertices $v_{j}$ are of degree 1 , operations $\left(\mathrm{K}_{i}\right), i=1,2,3$, can be obtained by composition of operations (B), (D) and (R). 
Table 1. Elementary deformations of a labeled Reeb graph
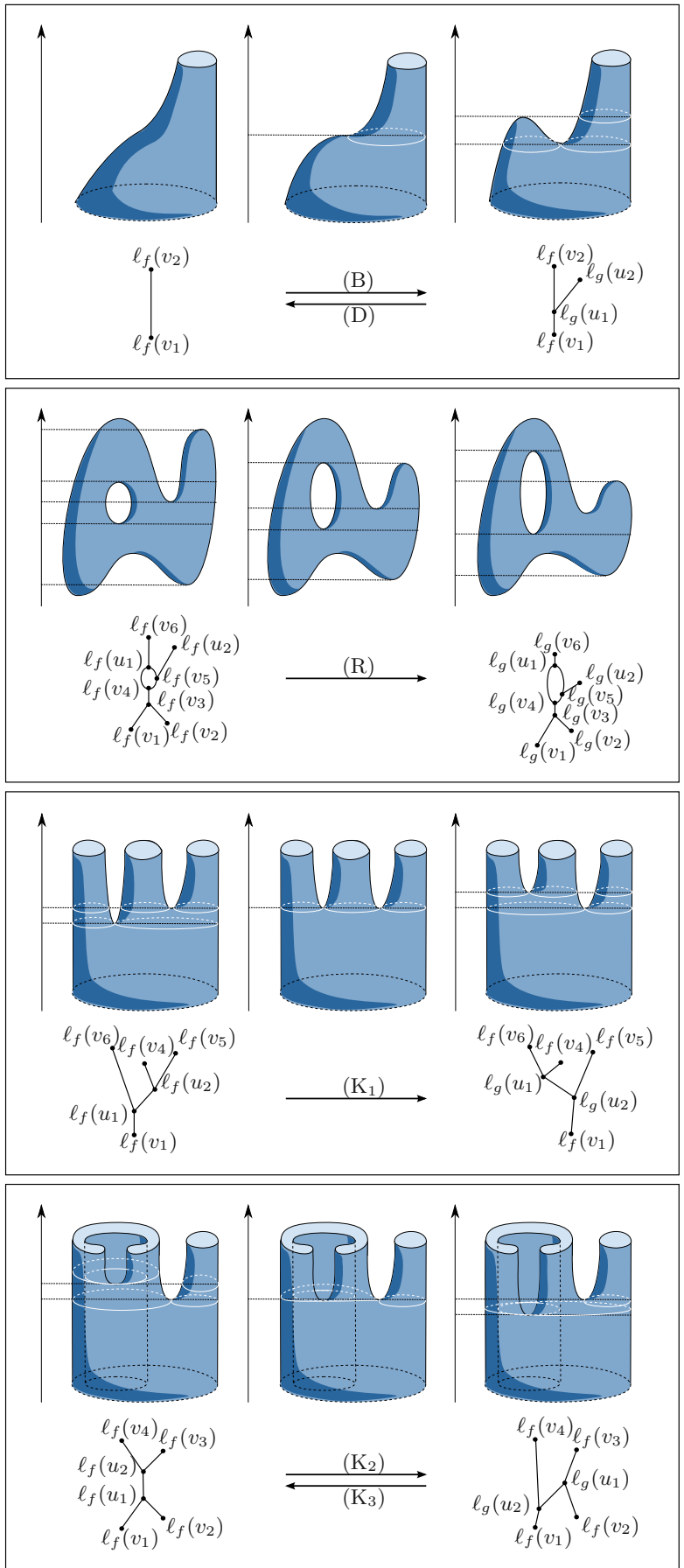
Since each type of elementary deformation transforms a labeled Reeb graph into another one, we can apply elementary deformations iteratively to transform labeled Reeb graphs into each other.

Definition 5. We shall call deformation of $\left(\Gamma_{f}, \ell_{f}\right)$ any finite ordered sequence $T=\left(T_{1}, T_{2}, \ldots, T_{r}\right)$ of elementary deformations such that $T_{1}$ is an elementary deformation of $\left(\Gamma_{f}, \ell_{f}\right), T_{2}$ is an elementary deformation of $T_{1}\left(\Gamma_{f}, \ell_{f}\right), \ldots$, $T_{r}$ is an elementary deformation of $T_{r-1} T_{r-2} \cdots T_{1}\left(\Gamma_{f}, \ell_{f}\right)$. We shall denote by $T\left(\Gamma_{f}, \ell_{f}\right)$ the result of the deformation $T$ applied to $\left(\Gamma_{f}, \ell_{f}\right)$. Moreover, we shall call identical deformation any deformation such that $T\left(\Gamma_{f}, \ell_{f}\right) \cong\left(\Gamma_{f}, \ell_{f}\right)$.

Proposition 3. Let $\left(\Gamma_{f}, \ell_{f}\right)$ and $\left(\Gamma_{g}, \ell_{g}\right)$ be two labeled Reeb graphs associated with simple Morse functions $f, g: \mathcal{M} \rightarrow \mathbb{R}$. Then the set of all the deformations $T$ such that $T\left(\Gamma_{f}, \ell_{f}\right) \cong\left(\Gamma_{g}, \ell_{g}\right)$ is non-empty.

In other words, any two Reeb graphs of simple Morse functions on a given surface can be transformed into each other by a finite sequence of elementary deformations. This result is a consequence of the fact that, through a finite sequence of elementary deformations of type (B), (D), (R), $\left(\mathrm{K}_{i}\right), i=1,2,3$, every Reeb graph can be transformed into one having only one maximum, one minimum, and all the cycles, if any, of length 2 [7].

\section{Editing Distance}

Given two labeled Reeb graphs $\left(\Gamma_{f}, \ell_{f}\right)$ and $\left(\Gamma_{g}, \ell_{g}\right)$ associated with simple Morse functions $f, g: \mathcal{M} \rightarrow \mathbb{R}$, we denote by $\mathcal{T}\left(\left(\Gamma_{f}, \ell_{f}\right),\left(\Gamma_{g}, \ell_{g}\right)\right)$ the set of all possible deformations between $\left(\Gamma_{f}, \ell_{f}\right)$ and $\left(\Gamma_{g}, \ell_{g}\right)$. Let us associate a cost with each editing deformation in $\mathcal{T}\left(\left(\Gamma_{f}, \ell_{f}\right),\left(\Gamma_{g}, \ell_{g}\right)\right)$.

Definition 6. Let $T$ be an elementary deformation such that $T\left(\Gamma_{f}, \ell_{f}\right) \cong\left(\Gamma_{g}, \ell_{g}\right)$.

- If $T$ is of type (B) inserting the vertices $u_{1}, u_{2} \in V\left(\Gamma_{g}\right)$, then we define the associated cost as

$$
c(T)=\frac{\left|\ell_{g}\left(u_{1}\right)-\ell_{g}\left(u_{2}\right)\right|}{2} .
$$

- If $T$ is of type (D) deleting the vertices $u_{1}, u_{2} \in V\left(\Gamma_{f}\right)$, then we define the associated cost as

$$
c(T)=\frac{\left|\ell_{f}\left(u_{1}\right)-\ell_{f}\left(u_{2}\right)\right|}{2} .
$$

- If $T$ is of type (R) relabeling the vertices $v \in V\left(\Gamma_{f}\right)=V\left(\Gamma_{g}\right)$, then we define the associated cost as

$$
c(T)=\max _{v \in V\left(\Gamma_{f}\right)}\left|\ell_{f}(v)-\ell_{g}(v)\right| .
$$

- If $T$ is of type $\left(\mathrm{K}_{i}\right)$, with $i=1,2,3$, relabeling the vertices $u_{1}, u_{2} \in V\left(\Gamma_{f}\right)$, then we define the associated cost as

$$
c(T)=\max \left\{\left|\ell_{f}\left(u_{1}\right)-\ell_{g}\left(u_{1}\right)\right|,\left|\ell_{f}\left(u_{2}\right)-\ell_{g}\left(u_{2}\right)\right|\right\} .
$$


Moreover, if $T=\left(T_{1}, \ldots, T_{r}\right)$ is a deformation such that $T_{r} \cdots T_{1}\left(\Gamma_{f}, \ell_{f}\right) \cong$ $\left(\Gamma_{g}, \ell_{g}\right)$, we define the associated cost as $c(T)=\sum_{i=1}^{r} c\left(T_{i}\right)$.

Now we can define the editing distance between labeled Reeb graphs as the infimum cost we have to bear to transform one graph into the other [7, Thm. $3.3]$.

Theorem 1. For every two labeled Reeb graphs $\left(\Gamma_{f}, \ell_{f}\right)$ and $\left(\Gamma_{g}, \ell_{g}\right)$, we set

$$
d\left(\left(\Gamma_{f}, \ell_{f}\right),\left(\Gamma_{g}, \ell_{g}\right)\right)=\inf _{T \in \mathcal{T}\left(\left(\Gamma_{f}, \ell_{f}\right),\left(\Gamma_{g}, \ell_{g}\right)\right)} c(T) .
$$

Then $d$ is a pseudo-metric on isomorphism classes of labeled Reeb graphs.

We recall that a pseudo-metric is non-negative, symmetric, and has the triangle inequality, but may be unable to distinguish different objects.

We do not exclude that our editing distance may have also the coincidence axiom as in the case of curves. If so, it would turn to be a metric. The main difficulty is that the linearization technique used in the case of curves does not work in the case of surfaces. We are currently investigating different techniques.

\section{$5 \quad$ Stability Result}

Let $\mathcal{F}(\mathcal{M}, \mathbb{R})$ be the set of smooth real valued functions on $\mathcal{M}$, endowed with the $C^{\infty}$ topology, and let us stratify such a space, as done by Cerf in [5]. Let us denote by $\mathcal{F}^{0}$ the submanifold of $\mathcal{F}(\mathcal{M}, \mathbb{R})$ of co-dimension 0 that contains all the simple Morse functions $f: \mathcal{M} \rightarrow \mathbb{R}$. Then, let $\mathcal{F}^{1}=\mathcal{F}_{\alpha}^{1} \cup \mathcal{F}_{\beta}^{1}$ be the submanifold of $\mathcal{F}(\mathcal{M}, \mathbb{R})$ of co-dimension 1 , where: $\mathcal{F}_{\alpha}^{1}$ represents the set of functions whose critical levels contain exactly one critical point, and the critical points are all non-degenerate, except exactly one; $\mathcal{F}_{\beta}^{1}$ the set of Morse functions whose critical levels contain at most one critical point, except for one level containing exactly two critical points.

The main result, proven in [7], is the following one.

Theorem 2 (Stability Theorem). For every $f, g \in \mathcal{F}^{0}$,

$$
d\left(\left(\Gamma_{f}, \ell_{f}\right),\left(\Gamma_{g}, \ell_{g}\right)\right) \leq\|f-g\|_{C^{0}},
$$

where $\|f-g\|_{C^{0}}=\max _{p \in \mathcal{M}}|f(p)-g(p)|$.

The proof relies on two intermediate results. The first one states that, considering a linear path connecting two functions $f, g \in \mathcal{F}^{0}$ and not traversing strata of co-dimension greater than 0 , the editing distance between the Reeb graphs associated with its end-points is upper bounded by the distance of $f$ and $g$ computed in the $C^{0}$-norm. In this case the graph $\left(\Gamma_{g}, \ell_{g}\right)$ can be obtained transforming $\left(\Gamma_{f}, \ell_{f}\right)$ with a sequence of elementary deformations of type $(\mathrm{R})$. 
- Let $f, g \in \mathcal{F}^{0}$ and let us consider the path $h:[0,1] \rightarrow \mathcal{F}(\mathcal{M}, \mathbb{R})$ defined by $h(\lambda)=(1-\lambda) f+\lambda g$. If $h(\lambda) \in \mathcal{F}^{0}$ for every $\lambda \in[0,1]$, then $d\left(\left(\Gamma_{f}, \ell_{f}\right),\left(\Gamma_{g}, \ell_{g}\right)\right) \leq\|f-g\|_{C^{0}}$.

The second result states that, if two functions $f, g \in \mathcal{F}^{0}$ can be connected by a linear path having only one point which belong to a stratum $\mathcal{F}^{1}$ and do not traverse strata of co-dimension greater than 1 , the cost to transform $\left(\Gamma_{f}, \ell_{f}\right)$ into $\left(\Gamma_{g}, \ell_{g}\right)$ is again upper bounded by $\|f-g\|_{C^{0}}$. In particular, crossing a stratum $\mathcal{F}_{\alpha}^{1}\left(\mathcal{F}_{\beta}^{1}\right.$, resp. $)$, means that the Reeb graph is undergoing an elementary deformation of type (B) or (D) ((R) or $\left(\mathrm{K}_{i}\right), i=1,2,3$, resp.).

- Let $f, g \in \mathcal{F}^{0}$ and let us consider the path $h:[0,1] \rightarrow \mathcal{F}(\mathcal{M}, \mathbb{R})$ defined by $h(\lambda)=(1-\lambda) f+\lambda g$. If $h(\lambda) \in \mathcal{F}^{0}$ for every $\lambda \in[0,1] \backslash\{\bar{\lambda}\}$, with $0<\bar{\lambda}<1$, and $h$ transversely intersects $\mathcal{F}^{1}$ at $\bar{\lambda}$, then $d\left(\left(\Gamma_{f}, \ell_{f}\right),\left(\Gamma_{g}, \ell_{g}\right)\right) \leq\|f-g\|_{C^{0}}$.

As an example illustrating the stability property of the editing distance, consider $f, g: \mathcal{M} \rightarrow \mathbb{R}$ as in Figure 2 Let $f\left(q_{i}\right)-f\left(p_{i}\right)=a, i=1,2,3$. It holds that $d\left(\left(\Gamma_{f}, \ell_{f}\right),\left(\Gamma_{g}, \ell_{g}\right)\right) \leq \frac{a}{2}$, showing that the editing distance is bounded by the norm of the difference between the functions. Indeed, for every $0<\epsilon<\frac{a}{2}$, we can apply to $\left(\Gamma_{f}, \ell_{f}\right)$ a deformation of type $(\mathrm{R})$, that relabels the vertices $p_{i}, q_{i}, i=1,2,3$, in such a way that $\ell_{f}\left(p_{i}\right)$ is increased by $\frac{a}{2}-\epsilon$, and $\ell_{f}\left(q_{i}\right)$ is decreased by $\frac{a}{2}-\epsilon$, composed with three deformations of type (D) that delete $p_{i}$ with $q_{i}, i=1,2,3$. Thus, since the total cost is equal to $\frac{a}{2}-\epsilon+3 \epsilon$, by the arbitrariness of $\epsilon$, it holds that $d\left(\left(\Gamma_{f}, \ell_{f}\right),\left(\Gamma_{g}, \ell_{g}\right)\right) \leq \frac{a}{2}$.
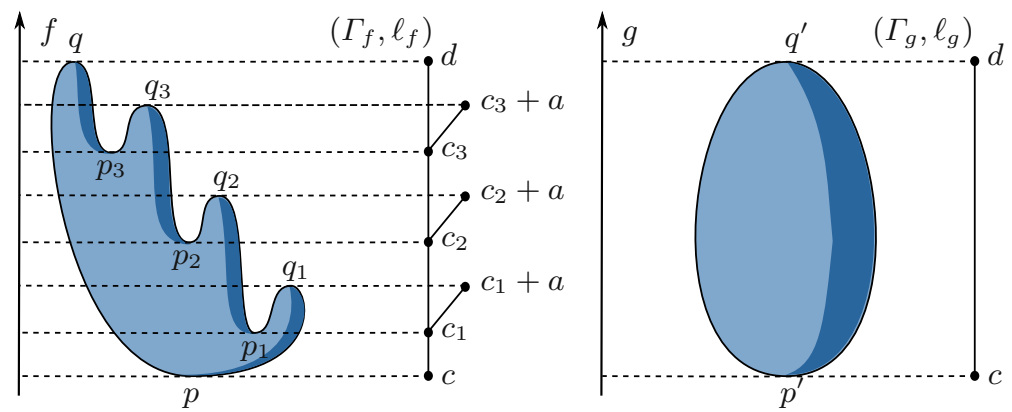

Fig. 2. For these two simple Morse functions $f, g$ it is easy to see that $d\left(\left(\Gamma_{f}, \ell_{f}\right),\left(\Gamma_{g}, \ell_{g}\right)\right)$ is bounded from above by the norm of $f-g$

\section{Discussion}

Building on arguments similar to those given in [6] for curves, we have presented a combinatorial dissimilarity measure for Reeb graphs of surfaces. For a complete analogy with the case of curves, we still need to prove that the editing distance is 
not only a pseudo-metric but actually a metric. Also, it would be useful to prove that, as for curves, it discriminates shapes as well as the natural pseudo-distance.

From the computational viewpoint, it would be very interesting to find an analogue of the editing distance for the case when the considered surfaces are discrete models, e.g. triangular meshes, and the functions accordingly discrete.

\section{References}

1. Bauer, U., Ge, X., Wang, Y.: Measuring distance between Reeb graphs. No. arXiv:1307.2839v1 (2013)

2. Beketayev, K., Yeliussizov, D., Morozov, D., Weber, G.H., Hamann, B.: Measuring the distance between merge trees. In: Topological Methods in Data Analysis and Visualization V (TopoInVis 2013) (2013)

3. Biasotti, S., Giorgi, D., Spagnuolo, M., Falcidieno, B.: Reeb graphs for shape analysis and applications. Theoretical Computer Science 392, 5-22 (2008)

4. Biasotti, S., Marini, S., Spagnuolo, M., Falcidieno, B.: Sub-part correspondence by structural descriptors of 3D shapes. Computer-Aided Design 38(9), 1002-1019 (2006)

5. Cerf, J.: La stratification naturelle des espaces de fonctions différentiables réelles et le théorème de la pseudo-isotopie. Inst. Hautes Études Sci. Publ. Math. 39, 5-173 (1970)

6. Di Fabio, B., Landi, C.: Reeb graphs of curves are stable under function perturbations. Mathematical Methods in the Applied Sciences 35(12), 1456-1471 (2012)

7. Di Fabio, B., Landi, C.: Reeb graphs of surfaces are stable under function perturbations. Tech. Rep. 3956, Università di Bologna (February 2014), http://amsacta.cib.unibo.it/3956/

8. Donatini, P., Frosini, P.: Natural pseudodistances between closed manifolds. Forum Mathematicum 16(5), 695-715 (2004)

9. Gao, X., Xiao, B., Tao, D., Li, X.: A survey of graph edit distance. Pattern Anal. Appl. 13(1), 113-129 (2010)

10. Hilaga, M., Shinagawa, Y., Kohmura, T., Kunii, T.L.: Topology matching for fully automatic similarity estimation of 3D shapes. In: ACM Computer Graphics (Proc. SIGGRAPH 2001), pp. 203-212. ACM Press, Los Angeles (2001)

11. Kudryavtseva, E.A.: Reduction of Morse functions on surfaces to canonical form by smooth deformation. Regul. Chaotic Dyn. 4(3), 53-60 (1999)

12. Ling, H., Okada, K.: An efficient earth mover's distance algorithm for robust histogram comparison. IEEE Transactions on Pattern Analysis and Machine Intelligence 29, 840-853 (2007)

13. Masumoto, Y., Saeki, O.: A smooth function on a manifold with given reeb graph. Kyushu J. Math. 65(1), 75-84 (2011)

14. Ohbuchi, R., Takei, T.: Shape-similarity comparison of 3D models using alpha shapes. In: 11th Pacific Conference on Computer Graphics and Applications, pp. 293-302 (2003)

15. Reeb, G.: Sur les points singuliers d'une forme de Pfaff complétement intégrable ou d'une fonction numérique. Comptes Rendus de L'Académie ses Sciences 222, 847-849 (1946) 
16. Shinagawa, Y., Kunii, T.L.: Constructing a Reeb Graph automatically from cross sections. IEEE Computer Graphics and Applications 11(6), 44-51 (1991)

17. Shinagawa, Y., Kunii, T.L., Kergosien, Y.L.: Surface coding based on morse theory. IEEE Computer Graphics and Applications 11(5), 66-78 (1991)

18. Wu, H.Y., Zha, H., Luo, T., Wang, X.L., Ma, S.: Global and local isometryinvariant descriptor for 3D shape comparison and partial matching. In: 2010 IEEE Conference on Computer Vision and Pattern Recognition (CVPR), pp. 438-445 (2010) 